\title{
Piomiositis: presentación de 17 casos en niños y adultos
}

\author{
lago Villamil-Cajoto ${ }^{1}$, Francisco Maceiras-Pan², \\ María José Villacián-Vicedo ${ }^{3}$.
}

\author{
Pyomyositis. Report of \\ seventeen cases
}

Background: Pyomyositis is a bacterial infection of skeletal muscle. Although more commonly seen in the tropics, it is increasingly recognized in temperate regions. The age distribution for patients with so called "tropical" or "temperate" pyomyositis differs. Most cases of tropical pyomyositis are seen in otherwise healthy patients and mainly in children, while the majority of cases of temperate pyomyositis occur in inmunocompromised adults. Aim: To report a series of patients with pyomyositis. Material and Methods: Retrospective review of clinical records of patients admitted to our hospital with pyomyositis during the period 1996-2001. Results: Seventeen patients were identified, aged from 5 to 86 years old, nine (53\%) males. Staphylococcus aureus (13 cases, 76\%) was the most common infecting organism. Eleven patients (65\%) had a history of previous trauma. All patients were immunocompetent. Six patients underwent surgical drainage. Six patients (35\%) presented complications and of those, one died. Conclusions: All patients of this series were immunocompetent. Pyomyositis is a serious and life threatening disease but curable. An early treatment is the key to a better prognosis (Rev Méd Chile 2006; 134: 31-38).

(Key words: Myositis; Pyomyositis, non tropical; Staphylococcus aureus)

Recibido el 26 de enero, 2005. Aceptado el 17 de junio, 2005.

${ }^{1}$ Servicio de Medicina Interna, ${ }^{2}$ Servicio de Reumatología, ${ }^{3}$ Servicio de Pediatría, Hospital Clínico Universitario de Santiago de Compostela. Choupana sn. Santiago de Compostela 15706 A Coruña. España.

$\mathrm{L}$ a piomiositis es una afección piógena del músculo estriado. Documentada por primera vez como serie de casos por Scriba en $1885^{1}$, se interpretó en su descripción como una enfermedad endémica de climas templados, calificándose inicialmente como «ropical». La descripción de casos en clima no tropical es más reciente ${ }^{2-4}, \mathrm{y}$

Correspondencia a: Dr. Iago Villamil Cajoto. Servicio de Medicina Interna, Hospital Clínico Universitario de Santiago de Compostela. Choupana sn, Santiago de Compostela 15706 A Coruña. España. Teléfono: 981959522. Fax: 981950501. E mail: iago.villamil.cajoto@sergas.es han sido descritas series pequeñas $\mathrm{y}$, fundamentalmente, casos aislados. Desde hace unos años $\mathrm{y}$, en posible relación con inmunodepresión nosocomial o adquirida, se describen series más amplias $^{5-8}$. En Europa existe creciente interés, estando documentados varios casos y algunas series hasta el momento ${ }^{9,10}$. Aunque se supone está determinada por sobreinfección bacteriana de un músculo predispuesto, su patogénesis no está bien determinada, y se postulan traumatismos, alteraciones nutricionales e infecciones virales entre otros, como posibles cofactores etiológicos $2,3,11$. Las diferencias iniciales más 
importantes entre las dos variantes (tropical y no tropical), se referían a las edades, número y áreas de músculos afectados ${ }^{3,4,12}$. En estudios recientes, tales diferencias no se aprecian, aunque se siguen describiendo los grupos tropical y no tropical. Otros factores de riesgo identificados se resumen en la Tabla $1^{9,13-18}$. Parece que en áreas no tropicales, la infección por virus de la inmunodeficiencia humana (VIH) sería determinante en su incidencia ${ }^{19}$. Desde 1988, las primeras descripciones apuntaban a una mayor incidencia en esta población con infección por $\mathrm{VIH}$, asociándose mayoritariamente con estadios avanzados de inmunodeficiencia (en pocos casos existía antecedentes de traumatismos), miositis por antirretrovirales (especialmente AZT) sin presentarse los factores de riesgo descritos y debidas a Staphylococcus $\mathrm{spp}^{7,15,18}$. La alta prevalencia como portador de Staphylococcus spp en el paciente con infección por VIH, podría explicar la mayor incidencia descrita de bacteriemia y piomiositis en estos pacientes ${ }^{20,21}$. Los factores por los que estaría aumentada la incidencia en pacientes con infección por VIH se resumen en la Tabla $2^{7,21-24}$.

Staphylococcus aureus (Saureus) es el microorganismo predominante tanto en inmunodeprimidos como inmunocompetentes ${ }^{12,14}$. Sin embargo, se

Tabla 1. Factores de riesgo de Piomiositis, ${ }^{9,12-17}$

- Infección VIH

- Daño Muscular

- Ejercicio intenso

- Otros grados de inmunocompromiso:

- Cualquier causa de neutropenia e Inmunodeficiencia

- Uso de corticoterapia

- Agammaglobulinemia

- Anemia aplástica

- Uso de drogas intravenosas

- Infección por parásitos

- Déficit nutricional

- Miositis viral

- Leptospirosis

- Diabetes mellitus

- Hepatopatía alcohólica

- Leucemia

- Esclerodermia documentan nuevos microorganismos de forma cada vez más frecuente en los casos de piomiositis no tropical y en los casos asociados a pacientes con infección por $\mathrm{VIH}^{15-18}$. Clínicamente, se puede dividir en 3 estadios clínicos ${ }^{12}$ (Tabla 3) y afecta típicamente músculos largos estriados como los de pierna, caderas y región glútea. Los datos clínicos de la piomiositis tropical y no tropical son indistinguibles.

Existe, de acuerdo con lo expuesto, una permanente actualización de la enfermedad, que parece ir produciéndose a medida que se publican nuevas series. Hay, desde la década 1960-69, algo más de 676 casos descritos en la literatura incluida en bases de datos anglosajonas ${ }^{22}$. En el presente trabajo presentamos una serie amplia con datos actualizados que pretende contribuir a dar más información sobre esta enfermedad poco conocida y a establecer cada vez con más casuísti$\mathrm{ca}$, la ausencia de diferencias entre las llamadas piomiositis tropical y no tropical.

\section{PaCIENTES y MÉTOdos}

Se realizó el estudio retrospectivo de los pacientes diagnosticados de piomiositis en nuestro centro hospitalario en el período entre enero de 1996 y diciembre de 2001. Se trata de un hospital de tercer nivel, con 900 camas de hospitalización, que atiende un área sanitaria de 400.000 pacientes. La selección de casos se realizó a través del Servicio de Codificación del Hospital mediante

\section{Tabla 2. Factores que incrementan riesgo de piomiositis en VIH 7,21-26}

\footnotetext{
- Supresión de la inmunidad mediada por células T;

- Disfunción de linfocitos B y neutrófilos;

- Neutropenia secundaria a infiltración de médula ósea por distintas infecciones diseminadas;

- Defectos en la quimiotaxis y disminución de la capacidad bactericida de los PMN; aumento de la colonización por Staphylococcus aureus.

- Miopatías en relación a fármacos (zidovudina).

- Miositis por micobacterias.

- Elevación notable de concentraciones séricas de IgG en la enfermedad avanzada.
} 
Tabla 3. Estadios de piomiositis ${ }^{12}$

\begin{tabular}{|c|c|c|c|}
\hline & Clínica & Analítica & Evolución \\
\hline $\begin{array}{l}\text { Estadio } 1 \\
\text { ( } 2 \% \text { de los } \\
\text { casos) }\end{array}$ & $\begin{array}{l}\text { - Calambres musculares } \\
\text { locales, dolorosos, } \\
\text { inflamación, febrícula. } \\
\text { - Músculo moderadamente } \\
\text { indurado, no fluctúa. }\end{array}$ & $\begin{array}{l}\text { - Leucocitosis } \\
\text { ocasional } \\
\text { - Difícil extracción de } \\
\text { material purulento }\end{array}$ & \\
\hline $\begin{array}{l}\text { Estadio } 2 \\
(90 \% \text { de } \\
\text { los casos })\end{array}$ & $\begin{array}{l}\text { - Fiebre } \\
\text { - Fláccidez muscular }\end{array}$ & $\begin{array}{l}\text { - Leucocitosis marcada } \\
\text { Eosinofilia (tropical) } \\
\text { - Drenaje purulento }\end{array}$ & $\begin{array}{l}10 \text { días tras } \\
\text { inicio de } \\
\text { síntomas }\end{array}$ \\
\hline Estadio 3 & $\begin{array}{l}\text { - Sepsis } \\
\text { Músculo fluctuante }\end{array}$ & & $\begin{array}{l}\text { - Bacteriemia } \\
\text { Complicaciones }\end{array}$ \\
\hline
\end{tabular}

selección de diagnósticos codificados. Se incluyeron exclusivamente piomiositis primarias, esto es, no debidas a heridas penetrantes, úlceras por decúbito o extensión desde lugares vecinos (piomiositis secundaria). Al diagnóstico de piomiositis se llegó tras evaluar los resultados de imágenes descritos en los resultados, y el resultado de los cultivos de microorganismos. Se evaluaron todos los servicios hospitalarios incluyendo Pediatría, revisando las historias clínicas de los pacientes y los resultados de la pruebas microbiológicas. La incubación de los hemocultivos se realizó en un sistema automatizado de monitorización continua BacT/Alert (OrganonTecnica) durante un período de 5 días. A todos los hemocultivos positivos se les realizó una tinción de Gram y se subcultivaron en distintos medios de cultivo (agar sangre, agar chocolate, agar enriquecido) a $37^{\circ} \mathrm{C}$ en diferentes atmósferas (aerobia, microaerófila y anaerobia). La identificación de los aislamientos se realizó en el sistema autoanalizador Vitek2 (bioMérceux) siguiendo las instrucciones del fabricante. Los estudios de sensibilidad fueron determinados mediante el test de difusión disco-placa (método Bauer-Kirby) de acuerdo con los estándares definidos por el fabricante. En los procedimientos diagnósticos invasivos (punción de la lesión o drenaje quirúrgico), se realizó inicialmente tinción de Gram y cultivo de la muestra en medio aerobio y anaerobio así como cultivo para micobacterias.
Se describen las características epidemiológicas de los pacientes, la localización de la infección y los factores de riesgo asociados así como los tratamientos y evolución posteriores.

\section{RESULTAdos}

Se identificaron 17 pacientes diagnosticados de piomiositis primaria, de edades comprendidas entre 5 y 86 años. Nueve de ellos varones (52,9\%). Las características epidemiológicas, localizaciones y factores de riesgo asociados en cada caso están descritos en la Tabla 4. En total, 3 pacientes fueron clasificados como estadio 2 y 8 pacientes fueron incluidos en estadio 3. El tiempo de demora entre el inicio de los síntomas y el diagnóstico fue de media de 11 días (rango 6-21 días). En 14 pacientes (82,3\%) las lesiones se localizaron en las extremidades inferiores, 10 de ellos (77\%) presentan la lesión en la extremidad inferior izquierda (Figuras 1 y 2); un paciente presentó afección de ambos psoas ilíacos. En la mayoría de los pacientes $(13,76,5 \%)$ existía afección de un músculo exclusivamente. Los gérmenes más frecuentes fueron $\mathrm{S}$ aureus en 13 casos $(76,5 \%)$, oxacilin sensible 12 casos $(70,6 \%)$. En 2 pacientes no se identificó microorganismo $(11,8 \%)$; en éstos, la evolución clínica con tratamiento antibiótico fue buena inicialmente, por lo 


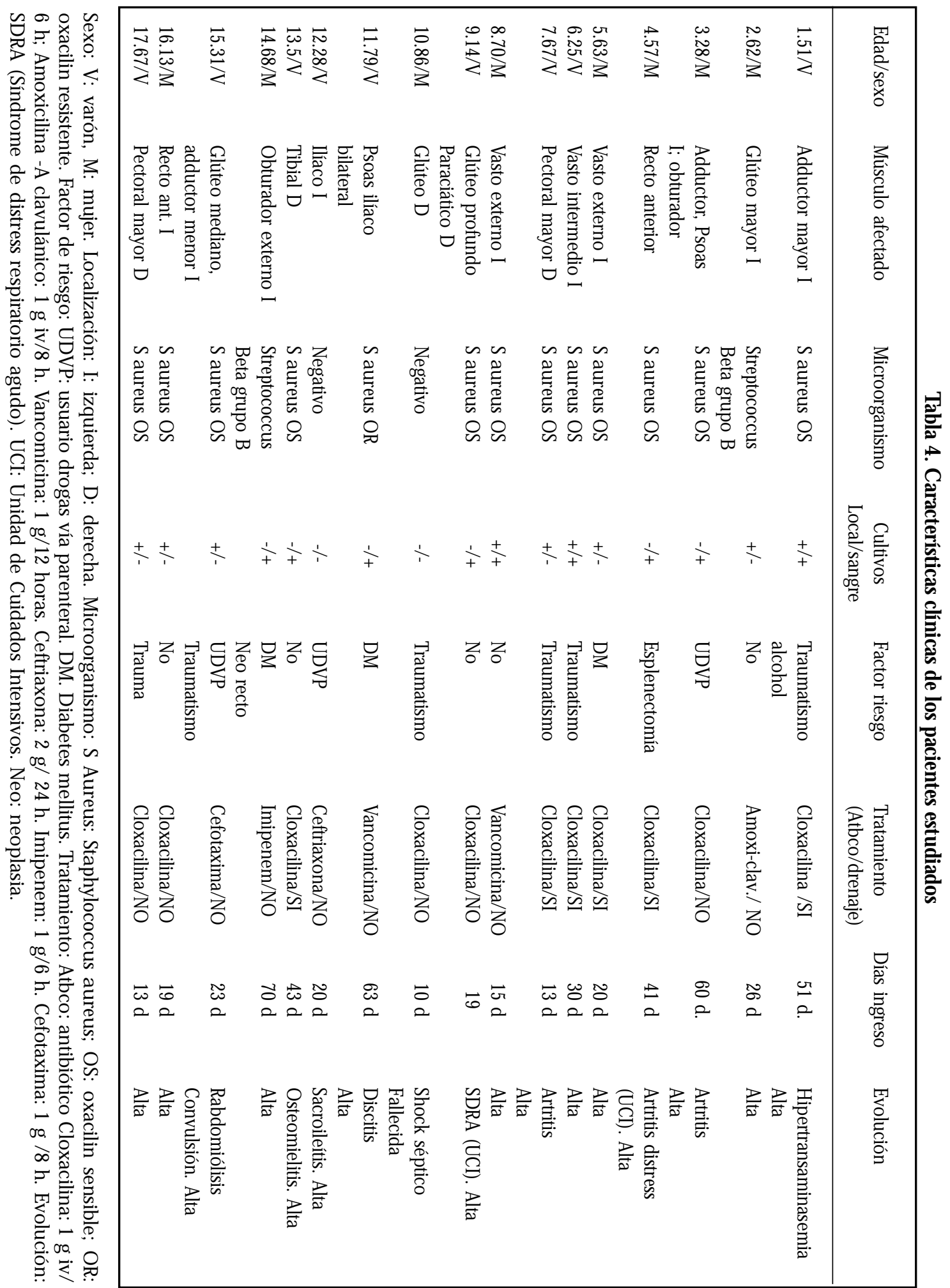


que se consideró en relación con proceso infeccioso bacteriano, aunque posteriormente presentaron complicaciones (uno, paciente 10, falleció y otro presentó sacroileítis). En todos los pacientes se realizó hemocultivos, en 9 pacientes (52,9\%) fueron positivos. Otras técnicas diagnósticas invasivas (punción del absceso o cirugía) fueron posibles en 14 pacientes (82,3\%), de ellas, 9 resultaron positivas $(64,2 \%)$. Se identificó traumatismo como posible desencadenante del proceso, en 11 pacientes $(64,7 \%)$. Once pacientes $(64,7 \%)$ presentaron complicaciones, 8 de ellos varones (72\%), incluyendo uno de los dos pacientes de edad pediátrica. Entre los pacientes con complicaciones, 3 de ellos (17\%), precisaron ingreso en unidad de cuidados intensivos (uno finalmente falleció; los otros dos fueron dados de alta posteriormente). Las complicaciones fueron: 4 artritis sépticas, 1 rabdomiólisis, 1 osteomielitis, 1 espondilodiscitis, 1 sacroileítis, 1 hepatitis en relación con bacteriemia, 1 episodio de distress respiratorio agudo, y un cuadro de shock séptico, que finalmente fue exitus (5\%). Ningún paciente presentaba inmunodeficiencia adquirida de origen infeccioso ni por citostáticos y, entre los posibles factores de riesgo asociados a la complicación, 3 pacientes $(17,6 \%)$ presentaban Diabetes Mellitus (DM), 2 eran usuarios de drogas por vía parenteral (UDVP) y uno de ellos presentaba una flebitis por

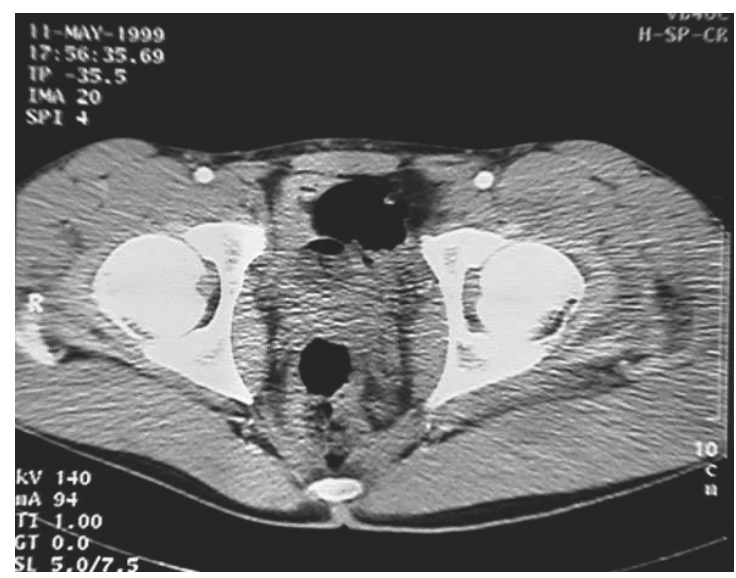

FiguRa 1. TC abdómino pélvico (paciente 15). Hipodensidad y borrosidad marcada que afecta fundamentalmente al glúteo mediano izquierdo. No se aprecian áreas de gas. un ingreso previo. En 2 de los pacientes con complicaciones graves (distress respiratorio agudo en un caso y shock séptico y exitus en el otro) no existía enfermedad de base alguna. En cuanto al tratamiento, todos los pacientes recibieron antibioterapia intravenosa desde la confirmación del cuadro infeccioso durante, al menos, 21 días. Once pacientes $(64,7 \%)$ recibieron cloxacilina con o sin asociación de aminoglucósidos, 1 paciente amoxicilina clavulánico, 2 pacientes cefalosporinas de 3 a generación, 1 paciente imipenem y 2 pacientes vancomicina (uno de ellos por ser el patógeno asilado oxacilin resistente y en el otro caso por presentar toxicodermia en relación con cloxacilina). En 6 pacientes (35\%) se realizó drenaje quirúrgico. La estancia media fue de 31,41 días, con un rango de entre 10 y 70 días. No hubo recidivas en el seguimiento posterior a los pacientes.

\section{DisCUSIÓN}

La piomiositis es una infección bacteriana del músculo estriado con escasa incidencia en Europa, aunque con una trascendencia en cuanto a morbimortalidad que debe tenerse en cuenta. Supone entre 1 y $4 \%$ de los ingresos hospitalarios en alguna área tropical ${ }^{27}$. La importancia del entorno inme-

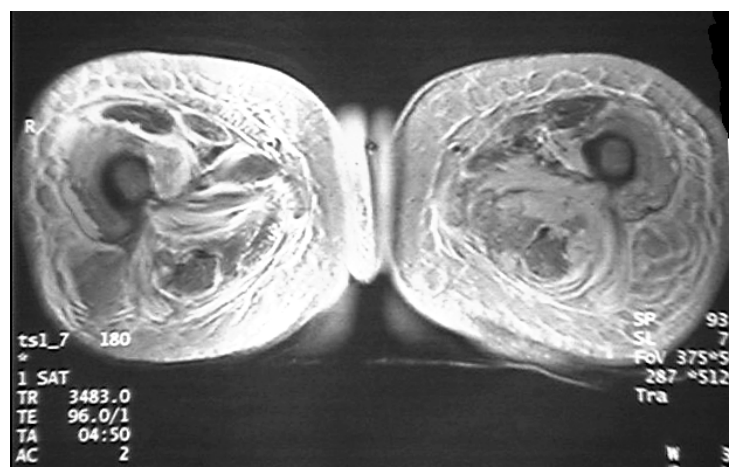

FIgURA 2. RM pélvica (paciente 14). Colección hipodensa en músculo obturador izquierdo. 
diato del músculo se trató de poner en evidencia en estudios experimentales sobre osteomielitis y piomiositis ${ }^{26,27}$. Algunos autores asumen que la invasión del músculo ocurre después de que una bacteriemia transitoria, asiente sobre una zona de la estructura muscular dañada previamente. Así, la infección desde el tracto gastrointestinal, urinario, respiratorio o desde catéteres intravasculares, puede predisponer a bacteriemia con piomiositis secundaria ${ }^{9}$. De forma clásica y en una revisión reciente $^{22}$, los pacientes afectos son varones jóvenes sanos, en los que al localizarse el absceso muscular bajo la fascia, se presenta de forma subaguda al ser el eritema, edema y calor mínimos hasta la extensión a tejido subcutáneo, días 0 semanas después. Las localizaciones más habituales son en miembros inferiores y músculos del tron$\mathrm{Co}^{10,12,28}$. Las diferencias iniciales más importantes entre las dos variantes (tropical y no tropical), se refería a las edades, número y áreas de músculos afectados: pacientes adultos, afectando varios grupos musculares y sobre todo en piernas, en el tipo tropical y, por el contrario, en la no tropical, pacientes pediátricos, afección muscular única y de predominio en muslos $3,4,12,22,31$, el germen en todos los casos era mayoritariamente S aureus. En estudios recientes, tales diferencias no se aprecian, aunque se siguen describiendo los grupos tropical y no tropical, asumiendo una incidencia creciente en el grupo no tropical en relación a la infección por $\mathrm{VIH}^{19,22}$. En nuestra serie se presenta la distribución inversa, predominio de adultos y en las piernas aun siendo no tropical, afectando a varios grupos musculares. En Estados Unidos de Norteamérica (EE.UU) y África, entre 33 y $40 \%$ del total de los casos de piomiositis se ve en niños ${ }^{12,31}$, sin embargo, en nuestro estudio tan solo se presentó en 2 pacientes pediátricos (11\%). En todos los casos parece existir también un predominio en varones $^{31,32}$. Nuestros pacientes eran en su mayoría adultos (15 pacientes, $88 \%$ ), y presentaron una distribución bastante uniforme en cuanto a género, tanto en la edad adulta (52,9\% varones), como en los 2 pacientes pediátricos (50\%). El microorganismo implicado más frecuente es S aureus (50-95\% de los casos) tanto en climas templados como no templados 22,27,31,32. En nuestra serie, en 13 casos (76,5\%), uno de ellos oxacilin resistente. Los hemocultivos son positivos en menos de $5 \%$ de los casos en el momento de la presentación clínica, con un máximo de positividad de 35\% según las series ${ }^{12,27}$. En nuestros pacientes, la demostración microbiológica en sangre fue de 53\% (9 casos). El resto de los casos con germen conocido suele deberse a Streptococcus beta hemolítico grupo A y Streptococcus pyogenes ${ }^{12,22}$. Por tanto, la etiología en los pacientes presentados, es coincidente con lo publicado previamente. El tratamiento es, en la mayoría de los casos, quirúrgico ${ }^{3,31}$, entre nuestros pacientes el drenaje quirúrgico sólo se pudo realizar en 6 pacientes. En el resto no se pudo por dificultades técnicas, dada la localización, por negativa del paciente a la intervención, presentándose complicaciones graves en aquellos en que no fue posible el drenaje. Una limitación en nuestro estudio fue que la mayoría de los casos se presentan en una situación clínica equivalente a estadios avanzados por lo que el mal pronóstico y el índice de complicaciones podrían estar sesgados en este sentido. También es cierto que la realización de la técnica se produjo ante la evidencia de complicaciones (artritis) en 4 de los pacientes. De esta forma no se puede asumir que fueran peor los pacientes con drenaje. La adecuación del tratamiento al antibiograma del microorganismo se produjo en todos los casos, manteniéndose el antibiótico empírico en todos los pacientes, salvo en los dos casos de uso de vancomicina en que ante la presencia de S aureus oxacilin resistente en uno y por toxicodermia a cloxacilina en otro se cambia a glicopéptido.

Una característica de interés presente en nuestra serie fue la ausencia, en la mayoría de los casos, de factores de riesgo para la enfermedad. Se identificó traumatismo previo en 11 pacientes $(64,7 \%), 8$ de ellos varones que corresponde al perfil típico de las series de piomiositis tropical. En otras series, la aparición de factores de riesgo se da hasta en $40 \%$ de los $\operatorname{casos}^{27}$, en las no tropicales. En nuestra serie, se identificó la DM como factor de riesgo, aunque presente tan solo en 3 pacientes $(17,6 \%)$. Ningún paciente presentaba inmunodeficiencia; este dato es especialmente notable por la asociación a la infección por VIH que en la mayoría de las series de piomiositis no tropical, se identifica como factor de riesgo cla$\mathrm{ve}^{19}$. Es probable que los cambios en la situación de estos enfermos, tras la estandarización de la terapia antirretroviral de gran actividad, signifique menor incidencia de piomiositis. Ante esto, al 
igual que lo expuesto anteriormente, nos hace creer que nuestra serie contribuye a insistir en la ausencia de diferencias entre las dos supuestas entidades (piomiositis tropical y no tropical).

En definitiva, la piomiositis es una entidad importante, de trascendencia clínica notable en cuanto a morbimortalidad, con estancias medias prolongadas. Aunque no suponen porcentajes tan elevados de ingresos como los descritos en África, no es excepcional, por lo que debe prestarse atención continua a esta patología ${ }^{22}$. Además, el ascenso de esta patología que se presenta en climas no templados y que se relaciona funda-

\section{REFERENCIAS}

1. ScriBa J. Beitrag zur Aetiologie der Myositis acuta. Dtsch Z Chir 1885; 22: 497-502.

2. Geelhoed GW, Gray $\mathrm{H}$, Alavi iA, Lattoo $\mathrm{H}$. Pyomyositis-tropical and non tropical. N Engl J Med 1971; 284: 850-4.

3. Brown JD, Wheeler B. Pyomyositis: report of 18 cases in Hawaii. Arch Intern Med 1984; 144: 1749-51.

4. Gibson RK, Rosenthal SJ, Luker BP. Pyomyositisincreasing recognition in temperate climates. Am J Med 1984; 77: 768-72.

5. Caldwell DS, Kernodle GW JR, Seigler HF. Pectoralis pyomyositis: an unusual case of chest pain in a patient with diabetes mellitus and rheumatoid arthritis. J Rheumatol 1986; 13: 434-6.

6. Vartian C, Septimus EJ. Pyomyositis in an intravenous drug user with human inmunodeficiency virus. Arch Intern Med 1988; 148: 2689.

7. Swartzman WA, Lambertus MW, Kennedy CA, Goetz MB. Staphyloccal pyomyositis in patients infected by the human inmunodeficiency virus. Am J Med 1991; 90: 595-600.

8. BOWEn PA, WYNn JW, Fischer AQ, Howard AC, McMAHON J. Nontropical pyomyositis in a renal allograft recipient. Transplantation 1989; 48: 539-41.

9. Gómez-Reino JJ, Aznar JJ, Pablos JL, Díaz-González F, LAFFon A. Nontropical pyomyositis in adults. Semin Arthritis Rheum 1994; 23: 396-405.

10. Chimeno MM, Chocarro A, Arias M, Palomar LM, Rodríguez P, Villafane C et al. Piomiositis en Zamora. Enferm Infecc Microbiol Clin 1996; 14: 27-30. mentalmente con inmunodeficiencia, no parece exclusivamente relacionado con ésta como puede comprobarse por nuestros resultados. Las complicaciones en nuestra serie fueron mayores que en otros estudios, dada la importante prevalencia de estadios 2 y 3 de la enfermedad. Esto hace que la sospecha diagnóstica y el tratamiento precoz sea fundamental para una buena evolución ${ }^{9}$. Las diferencias epidemiológicas entre las formas tropical y no tropical son cada vez más difíciles de sostener. A medida que se publiquen más casos, creemos que desaparecerán confirmándose como una única entidad.

11. Traquir RN. Pyomyositis. J Trop Med Hyg 1947; 50: 81-9.

12. Chiedozi LC. Pyomyositis: Review of 205 cases in 112 patients. Am J Surg 1979; 137: 255-9.

13. Christin L, Sarosi GA. Pyomyositis in North America: Case reports and review. Clin Infect Dis 1992; 15: 668-77.

14. Skoutelis A, Andonopoulos A, Panagiotopoulos E. Non-tropical pyomyositis in adults: Report of four cases and literature review. Eur J Clin Microbiol Infect Dis 1993; 12: 769.

15. Widrow CA, Kewe SM, Saltzman BR, Mathur-Wegh U. Pyomyositis in patients with the human immunodeficiency virus: An unusual form of disseminated bacterial infection. Am J Med 1991; 91: 129-36.

16. Falasca GF, Reginato AJ. The spectrum of myositis and rhabdomyolysis associated with bacterial infection. J Rheumatol 1994; 21: 1932-7.

17. Hsuen PR, Hsiue TR, Hsien WC. Pyomyositis in intravenous drug abusers: Report of a unique case and review of the literature. Clin Infect Dis 1996; 22: 858-60.

18. Vassilopoulos D, Chalasanu P, Jurado RL, WorkowsKI K, Agudelo CA. Musculoskeletal infections in patients with human immunodeficiency virus infection. Medicine (Baltimore) 1997; 76: 284-94.

19. CRUM NF. Bacterial Pyomyositis in the United States. Am J Med 2004; 117: 420-8.

20. Raviglone MP, Pablos-Méndez A, Battan R. Staphylococcus aureus nasal colonization rate in patients with AIDS or ARC (Abstract MBP 72). Fifth International Conference on AIDS. Montreal 1989; 233. 
21. Ganesh R, Castle D, McGibbon D, Phiшups I, BRADBEER C. Staphylococcal carriage and HIV infection. Lancet 1989; 2: 558.

22. Bikels J, Ben-Sira L, Kessler A, Wientroub S. Primary pyomyositis. J Bone Joint Surg Am 2002; 84-A: 2277-86.

23. JimÉnez-Mejías ME, Lozano de León F, Alfaro-García MJ, Fernández-López A, Jiménez-Ocaña C, Canas Gracía-Otero E. Piomiositis por Staphylococcus aureus. Med Clin (Barc) 1992; 99: 201-5.

24. HenRy MB, David SS. Pyomyositis and human immunodeficiency virus infection. South Med J 1990; 83: 1092-8.

25. JEшs JE. Viral infections: musculoskeletal infection in the human immunodeficiency virus (HIV)-infected patient. Baillieres Clin Rheumatol 1995; 9: 121-32.

26. Rodgers WB, Yodlowski ML, Mintzer CM. Pyomyositis in patients who have the human immunodeficiency virus. Case report and review of the literature. J Bone Joint Surg Am 1993; 75: 588-92.
27. Swartz MN. Myositis, Pyomiositis. En Mandell GL, Douglas RG, Bennett JE, ed. Principles and Practice of Infectious Diseases, Fifth ed. Philadelphia: Churchill Livingstone INC; 2000; 1058-60.

28. MoRRISSY RT, HaYnes DW. Acute hematogenous osteomielitis: a model with trauma as an ethiology. J Pediatr Orthop 1989; 4: 447-56.

29. SMITH IM, VICKERS AB. Natural history of 338 treated and untreated patients with staphylococcal septicemia (1936-1955). Lancet 1960; 1: 131822.

30. Patel SR, Oleguinski TP, Perruquet JL, Harrington TM. Pyomiositis: Clinical features and predisposing conditions. J Rheumatol 1997; 24: 1734-8.

31. Gubbay AJ, Isaacs D. Pyomiositis in children. Pediatr Infect Dis J 2000; 19: 1009-13.

32. Spiegel DA, Meyer JS, Dormans JP, Flynn JM, Drummond DS. Pyomiositis in children and adolescents: report of 12 cases and review of the literature. J Pediatr Orthop 1999; 19: 143-50. 